PROCEEDINGS OF THE

AMERICAN MATHEMATICAL SOCIETY

Volume 139, Number 12, December 2011, Pages 4285-4291

S 0002-9939(2011)11222-X

Article electronically published on July 12, 2011

\title{
SOFIC REPRESENTATIONS OF AMENABLE GROUPS
}

\author{
GÁBOR ELEK AND ENDRE SZABÓ
}

(Communicated by Jonathan I. Hall)

\begin{abstract}
Using probabilistic methods, Collins and Dykema proved that the free product of two sofic groups amalgamated over a monotileabe amenable subgroup is sofic as well. We show that the restriction is unnecessary; the free product of two sofic groups amalgamated over an arbitrary amenable subgroup is sofic. We also prove a group-theoretical analogue of a result of Kenley Jung. A finitely generated group is amenable if and only if it has only one sofic representation up to conjugacy equivalence.
\end{abstract}

\section{INTRODUCTION}

First, let us recall the notion of sofic groups (see 8 for a survey). Let $\Gamma$ be a finitely generated group with a symmetric generating system $S$. Let $G$ be a finite graph such that each directed edge of $G$ is labeled by an element of $S$. We say that $G$ is an $r$-approximation of the Cayley graph of $\Gamma, \operatorname{Cay}(\Gamma, S)$ if there exists a subset $W \subseteq V(G)$ such that

- $|W|>\left(1-\frac{1}{r}\right)|V(G)|$ and

- if $p \in W$, then the $r$-neighborhood of $p$ is rooted isomorphic to the $r$ neighborhood of a vertex of the Cayley graph of $\Gamma$ (as edge labeled graphs).

The group $\Gamma$ is sofic if for any $r \geq 1$ there exist $r$-approximations of $\operatorname{Cay}(\Gamma, S)$ by finite graphs. We call a not necessarily finitely generated group sofic if all of its finitely generated subgroups are sofic. Amenable and residually finite groups are sofic. Free products, direct products, inverse and direct limits of sofic groups are all sofic groups as well. Amenable extensions of sofic groups are also sofic. It is important to note that no nonsofic group is known at this moment. Several conjectures about groups, such as the Gottschalk Conjecture, Kaplansky's Direct Finiteness Conjecture, the Determinant Conjecture and the Connes Embedding Conjecture hold for sofic groups.

Using probabilistic arguments, Collins and Dykema [1] proved that the free products of sofic groups amalgamated over monotileabe amenable groups are sofic. We show that the restriction is not necessary.

Theorem 1. Free products of sofic groups amalgamated over arbitrary amenable groups are sofic.

Received by the editors October 25, 2010.

2010 Mathematics Subject Classification. Primary 20F65.

Key words and phrases. Sofic groups, amenable groups, amalgamated products.

Research sponsored by OTKA Grants No. 69062 and NK 78439.

(C)2011 American Mathematical Society

Reverts to public domain 28 years from publication 
In [6], Kenley Jung gave a characterization of amenable von Neumann algebras among "embeddable" von Neumann algebras. He proved that an embeddable von Neumann algebra $M$ is amenable if and only if any two embeddings of $M$ into $R^{\omega}$ are conjugate by a unitary element. Before stating our result, let us briefly recall an equivalent definition of soficity. Let $\omega$ be a nonprincipal ultrafilter on the natural numbers and $\lim _{\omega}: l^{\infty}(\mathbb{N}) \rightarrow \mathbb{R}$ be the associated ultralimit. Consider the product of finite symmetric groups $\prod_{n=1}^{\infty} S_{n}$. If $g \in S_{n}$, then let the rank of $g$ be defined as $r k(g):=1-\frac{f i x(\gamma)}{n}$, where $f i x(g)$ is the number of elements in the set $\{1,2, \ldots, n\}$ fixed by the permutation $g$. Let $H \subset \prod_{n=1}^{\infty} S_{n}$ be defined as follows:

$$
H:=\left\{\left(g_{n}\right)_{n=1}^{\infty} \mid \lim _{\omega} r k(g)=0\right\} .
$$

Then $H$ is a normal subgroup of $\prod_{n=1}^{\infty} S_{n}$ and $\Sigma=\prod_{n=1}^{\infty} S_{n} / H$ is a simple group with a rank function

$$
r k(\underline{g})=\lim _{\omega} r k\left(q_{n}\right) .
$$

We call an embedding $\Gamma \rightarrow \Sigma$ faithful if $r k(\gamma)=1$ for any $1 \neq \gamma \in \Gamma$. It is easy to see that sofic approximations lead to faithful embeddings of countable sofic groups. Conversely, any embeddable group is sofic [5].

The embeddings $\phi$ and $\psi$ are conjugate if there exists $\underline{g} \in \Sigma$ such that $\phi(\gamma)=$ $g \psi(\gamma) g^{-1}$ for any $\gamma \in \Gamma$. We prove the following theorem.

Theorem 2. A finitely generated group $\Gamma$ is amenable if and only if any two faithful embeddings of $\Gamma$ into $\Sigma$ are conjugate.

Note added. Liviu Paunescu [7] informed us that he also has a proof of Theorem 1. using a different argument.

\section{Sofic approximations of amenable groups}

Definition 2.1. Let $E(A)$ denote the set of edges in a colored graph $A$. We say that two colored graphs $A$ and $B$ are $r$-isomorphic for some $r>0$ if there are subgraphs $A^{\prime} \subseteq A$ and $B^{\prime} \subseteq B$ such that

$$
\left|E\left(A^{\prime}\right)\right| \geq\left(1-\frac{1}{r}\right)|E(A)|, \quad\left|E\left(B^{\prime}\right)\right| \geq\left(1-\frac{1}{r}\right)|E(B)|
$$

and $A^{\prime}$ is isomorphic to $B^{\prime}$ (as colored graphs). The isomorphism between $A^{\prime}$ and $B^{\prime}$ is called an $r$-isomorphism.

Lemma 2.2. Let $A, B$ and $C$ be colored graphs such that $A$ is $2 r$-isomorphic to $B$ and $B$ is $2 r$-isomorphic to $C$. Then $A$ is $r$-isomorphic to $C$. If $A$ is a $2 r$ approximation of a Cayley graph and every vertex of $A$ has degree at most $d$, then there is a bound $R_{\mathrm{appr}}(r, d)$ such that every colored graph $R_{\mathrm{appr}}(r, d)$-isomorphic to $A$ is an r-approximation of the Cayley graph.

Proof. This is clear from the definition.

Definition 2.3. Let $\mathcal{T}=T_{1}, T_{2}, \ldots, T_{m}$ be a finite sequence of colored graphs. Their linear combination with coefficient vector $\alpha=\left(\alpha_{1}, \alpha_{2}, \ldots, \alpha_{m}\right) \in \mathbb{N}^{m}$, denoted by $\alpha \cdot \mathcal{T}$, is the disjoint union of $\alpha_{1}$ copies of $T_{1}, \alpha_{2}$ copies of $T_{2}, \ldots$ and $\alpha_{m}$ copies of $T_{m}$. As a special case, if $m=1$, then we talk about the integer multiples of $T_{1}$.

Definition 2.4. For vectors $0 \neq \alpha \in \mathbb{R}^{m}$ we define the unit vector $\sigma(\alpha)=\frac{\alpha}{\|\alpha\|}$, where $\|\alpha\|$ denotes the (usual) length of $\alpha$. 
Lemma 2.5. Let $\mathcal{T}=\left\{T_{1}, T_{2}, \ldots, T_{m}\right\}$ be a finite sequence of colored graphs, each having at least one edge. For each integer $r>0$ there is an integer $M(r)>0$ (also depending on $\mathcal{T}$ ) such that whenever $\alpha, \beta \in \mathbb{N}^{m}$ are nonzero lattice vectors with $\|\beta\| \geq M(r)\|\alpha\|$ and $\|\sigma(\alpha)-\sigma(\beta)\| \leq \frac{1}{M(r)}$, then the graph $\beta \cdot \mathcal{T}$ is $r$-isomorphic to an integer multiple of $\alpha \cdot \mathcal{T}$.

Proof. Let $E$ be the maximum number of edges in $T_{i}$ and let $t$ be the largest integer such that $\|t \alpha\| \leq\|\beta\|$. Then $\|\alpha\| \geq 1$. Assuming that $M(r)$ as above exists, it will clearly satisfy the condition $M(r) \leq t$. Thus

$$
\|\beta-t \alpha\| \leq\|\alpha\|+t\|\sigma(\beta)-\sigma(\alpha)\| \leq\|\alpha\|+t \frac{1}{M(r)} \leq \frac{2}{M(r)}\|\alpha\| t
$$

hence each coordinate of $\beta$ differs by at most $\frac{2}{M(r)}\|\alpha\| t$ from the corresponding coordinate of $t \alpha$. Therefore $\beta \cdot \mathcal{T}$ can be obtained from $(t \alpha) \cdot \mathcal{T}$ by adding or deleting at most $\frac{2}{M(r)}\|\alpha\| t$ copies of each $T_{i}$, and the total number of edges involved in this operation is at most $\frac{2 m E}{M(r)}\|\alpha\| t$.

On the other hand, the sum of the coordinates of $t \alpha$ is at least $\|t \alpha\|$ (triangle inequality); hence the graph $t \alpha \cdot \mathcal{T}$ has at least $\|\alpha\| t$ edges, and similarly, the graph $\beta \cdot \mathcal{T}$ has at least $\|\beta\| \geq\|\alpha\| t$ edges. If we choose $M(r) \geq 2 m E r$, then we obtain that $\beta \cdot \mathcal{T}$ is $r$-isomorphic to $(t \alpha) \cdot \mathcal{T}$, which is indeed an integer multiple of $\alpha \cdot \mathcal{T}$.

Definition 2.6. Let $\Gamma$ be a finitely generated group, $S \subseteq \Gamma$ a finite symmetric generating set. A Følner sequence in the Cayley graph $\operatorname{Cay}(\Gamma, S)$ is a sequence $\mathcal{F}=\left\{F_{1}, F_{2}, \ldots\right\}$ of spanned subgraphs such that for all $r>0$ all but finitely many of the $F_{n}$ are $r$-approximations. The group $\Gamma$ is amenable if $\operatorname{Cay}(\Gamma, S)$ has a Følner sequence. Note, that this property depends only on the group; i.e. any other Cayley graph of $\Gamma$ has a Følner sequence. More generally, an arbitrary group is amenable if each of its finitely generated subgroups is amenable.

Proposition 2.7 (Reformulation of [2, Theorem 2]). Let $\Gamma$ be a finitely generated amenable group, $S \subseteq \Gamma$ a finite symmetric generating set and $\mathcal{F}$ a Følner sequence in $\operatorname{Cay}(\Gamma, S)$. Then for each integer $r>0$ there is an integer $R_{0}(r)>0$ (which depends also on $\mathcal{F}$ ) and a finite subsequence $\mathcal{T} \subset \mathcal{F}$ such that each $R_{0}(r)$-approximation of $\operatorname{Cay}(\Gamma, S)$ is r-equivalent to some linear combination of $\mathcal{T}$.

Proposition 2.8. Let $\Gamma$ be a finitely generated amenable group, $S \subseteq \Gamma$ a finite symmetric generating set and $\mathcal{F}$ a Følner sequence in $C a y(\Gamma, S)$. Then for each integer $r>0$ there is an integer $R_{\text {amenable }}(r)$ (which depends also on $\mathcal{F}$ ) and a finite subsequence $\mathcal{T} \subset \mathcal{F}$ of say $m$ members and a lattice vector $0 \neq \alpha \in \mathbb{N}^{m}$ with the following property. Each member of $\mathcal{T}$ is a 2 r-approximation of $C a y(\Gamma, S)$, and each $R_{\text {amenable }}(r)$-approximation of $C a y(\Gamma, S)$ is $2 r$-equivalent to an integer multiple of the (fixed) linear combination $\alpha \cdot \mathcal{T}$.

Proof. By discarding a few elements from $\mathcal{F}$ we may assume that the remaining elements are all $2 r$-approximations of $\operatorname{Cay}(\Gamma, S)$. We apply Proposition 2.7 with parameter $8 r$, and we obtain a subsequence $\mathcal{T} \subset \mathcal{F}$ of say $m$ members such that every $R_{0}(8 r)$-approximation of $\operatorname{Cay}(\Gamma, S)$ is $8 r$-isomorphic to some linear combination of $\mathcal{T}$. Now we discard those elements of $\mathcal{F}$ that are not an $R_{0}(8 r)$-approximation of $\operatorname{Cay}(\Gamma, S)$. Note that $\mathcal{T}$ is no longer a subsequence of the remaining $\mathcal{F}$.

For each member $F \in \mathcal{F}$ we choose a lattice vector $0 \neq \beta_{F} \in \mathbb{N}^{m}$ such that $\beta_{F} \cdot \mathcal{T}$ is $8 r$-isomorphic to $F$. Now $\left\{\sigma\left(\beta_{F}\right) \mid F \in \mathcal{F}\right\}$ is a sequence of unit vectors, 
so it has an accumulation point $u \in \mathbb{R}^{m}$. We apply Lemma 2.5 to $\mathcal{T}$ with parameter $8 r$ and obtain the bound $M(8 r)$. Let us fix a member $H \in \mathcal{F}$ such that $\left\|\sigma\left(\beta_{H}\right)-u\right\|<\frac{1}{2 M(8 r)}$ and then replace $\mathcal{F}$ by the subsequence of those $F \in \mathcal{F}$ that satisfy $\left\|\sigma\left(\beta_{F}\right)-u\right\|<\frac{1}{2 M(8 r)}$ and $\left\|\beta_{F}\right\| \geq M(8 r)\left\|\sigma\left(\beta_{H}\right)\right\|$. The remaining sequence $\mathcal{F}$ is still a $\mathrm{F} \varnothing$ lner sequence and for each $F \in \mathcal{F}$ we have $\left\|\sigma\left(\beta_{F}\right)-\sigma\left(\beta_{H}\right)\right\|<\frac{1}{M(8 r)}$; hence $\beta_{F} \cdot \mathcal{T}$ is $8 r$-isomorphic to an integer multiple of $\beta_{H} \cdot \mathcal{T}$ by Lemma 2.5

Applying again Lemma 2.7 with parameter $8 r$ we obtain a new finite subsequence $\left\{Q_{1}, Q_{2}, \ldots, Q_{n}\right\}$ of our modified $\mathcal{F}$. Let $X$ be any $R_{0}(8)$-approximation of $\operatorname{Cay}(\Gamma, S)$. Then $X$ is $8 r$-isomorphic to a linear combination of the $Q_{i}$, each $Q_{i}$ is $8 r$-isomorphic to the corresponding $\beta_{Q_{i}} \cdot \mathcal{T}$, and in turn each $\beta_{Q_{i}} \cdot \mathcal{T}$ is $8 r$ isomorphic to an integer multiple of $\beta_{H} \cdot \mathcal{T}$. Applying Lemma 2.2 twice we obtain that $X$ is $2 r$-isomorphic to an integer multiple of $\beta_{H} \cdot \mathcal{T}$. The lemma holds with $R_{\text {amenable }}(r)=R_{0}(8 r)$.

\section{Amalgamated products}

Definition 3.1. Let $\alpha$ and $\beta$ be partitions of a finite set $S$. The incidence graph of $\alpha$ and $\beta$ is a bipartite graph, whose two sets of vertices consist of the classes of $\alpha$ and the classes of $\beta$, and the edges are the elements of $S$, where each element connects its $\alpha$-class with its $\beta$-class.

Proposition 3.2 (proved in [4, Step 1]). For each triple $(a, b, r)$ of integers there is a finite set $S$ with two partitions $\alpha$ and $\beta$ on it such that each $\alpha$-class has a elements, each $\beta$-class has $b$ elements, an $\alpha$-class can meet a $\beta$-class in at most one element, and in the incidence graph of $\alpha$ and $\beta$ each simple cycle is longer than $2 r$.

Corollary 3.3. Let $\alpha, \beta$ and $S$ be as in Proposition 3.2. Suppose that the vertices of a graph are in $S$ and the endpoints of each edge are either in the same $\alpha$-class or in the same $\beta$-class. Then every simple cycle of length less than $2 r$ is either entirely in a single $\alpha$-class or entirely in a single $\beta$-class.

Proof. This follows immediately from Definition 3.1

Definition 3.4. Let $A$ be a colored graph and $Z$ a subset of the colors used in $A$. Then $\left.A\right|_{Z}$ denotes the subgraph obtained from $A$ by omitting all edges whose color does not belong to $Z$. Suppose that $C$ is another colored graph and $\phi$ is an $r$-isomorphism between $\left.A\right|_{Z}$ and $\left.C\right|_{Z}$. We build another graph called the

enhancement of $C$ with $A$ along $\phi$, denoted by $A \stackrel{\phi}{\Rightarrow} C$. We start from $C$ and add new edges to it. Namely, for each edge $a \rightarrow b$ of $A$ whose color does not belong to $Z$ we add a new edge $\phi(a) \rightarrow \phi(b)$ of the same color, provided that $\phi$ is defined at the endpoints $a$ and $b$.

Lemma 3.5. Let $\Gamma$ be a finitely generated group, $X$ be a finite symmetric generating system of $\Gamma$ and $Z \subseteq X$. Let $A$ be a $2 r$-approximation of $C a y(\Gamma, X)$ such that every vertex has degree at most $d$. Then there is a bound $R_{\mathrm{enh}}(r, d)$ with the following property. Suppose that $C$ is another colored graph with color set $Y$ such that $X \cap Y=$ $Z$ and $\phi$ is an $R_{\mathrm{enh}}(r, d)$-isomorphism between $\left.A\right|_{Z}$ and $\left.C\right|_{Z}$. Then $\left.(A \stackrel{\phi}{\Rightarrow} C)\right|_{X}$ is $r$-isomorphic to $A$ and $\left.(A \stackrel{\phi}{\Rightarrow} C)\right|_{Y}$ is isomorphic to $C$.

Proof. This is clear from the definition. 
Lemma 3.6. Let $G$ and $H$ be finitely generated sofic groups, $\Gamma \leq G$ a finitely generated amenable subgroup and $\phi: \Gamma \hookrightarrow H$ an injective homomorphism. Then the amalgamated product $G *_{\phi} H$ is also sofic.

Proof. For simplicity we identify both $G$ and $H$ with their canonical image in $G *_{\phi} H$, this will also identify both $\Gamma$ and $\phi(\Gamma)$ with $G \cap H$. Let $X \subseteq G, Y \subseteq H$ and $Z \subseteq \Gamma$ be finite symmetric generating systems such that $Z=X \cap Y$. Let $d$ be the maximum of $|X|$ and $|Y|$. The following is clear from the definition:

Claim 3.7. For each $r$ there is a constant $R_{\text {prod }}(r)$ with the following property. Suppose we find a colored graph $C$ with colors from $X \cup Y$ such that $\left.C\right|_{X}$ is an $R_{\text {prod }}(r)$ approximation of $\operatorname{Cay}(G, X),\left.C\right|_{Y}$ is an $R_{\text {prod }}(r)$-approximation of $\operatorname{Cay}(H, Y)$, and every simple cycle of length at most $2 r+1$ has either only $X$-colored edges or only $Y$-colored edges. Then $C$ is an $r$-approximation of the $\operatorname{Cay}\left(G *_{\phi} H, X \cup Y\right)$.

We choose any integer $r>0$ and set

$$
\begin{aligned}
& r_{1}=R_{\text {prod }}(r), \\
& r_{2}=R_{\text {appr }}\left(r_{1}, d\right), \\
& r_{3}=R_{\text {enh }}\left(r_{2}, d\right), \\
& r_{4}=R_{\text {amenable }}\left(r_{3}\right) .
\end{aligned}
$$

Let us start with $r_{4}$-approximations $A$, resp. $B$, of the Cayley graphs $C a y(G, X)$, resp. $C a y(H, Y)$. Then $\left.A\right|_{Z}$ and $\left.B\right|_{Z}$ are both $r_{4}$-approximations of $\operatorname{Cay}(\Gamma, Z)$. We choose a $\mathrm{F} \varnothing$ lner sequence in $\operatorname{Cay}(\Gamma, Z)$. Then Proposition 2.8 provides a colored graph $D$ (denoted there by $\alpha \cdot \mathcal{T}$ ) such that both $\left.A\right|_{Z}$ and $\left.B\right|_{Z}$ are $r_{3}$-isomorphic to integer multiples $a \cdot D$ and $b \cdot D$ (with appropriate integers $a, b>0$ ).

Applying Proposition 3.2 for the triple $(a, b, r+1)$ we obtain a finite set $S$ and partitions $\alpha$ and $\beta$ of $S$. Let $S_{0}$ denote the graph with no edge of vertex set $S$. Then the graph $C_{0}=S_{0} \times D$ is the union of $|S|$ disjoint copies of $D$, namely the subgraphs $\{s\} \times D$ for $s \in S$.

Now for each $\alpha$-class $\sigma \subset S$ the subgraph spanned by the subset $\sigma \times D$ is $r_{3}$-isomorphic to $\left.A\right|_{Z}$, so we can enhance it with $A$ along this $r_{3}$-isomorphism. Repeating this enhancement for all $\alpha$-classes we obtain a new graph $C_{1}$ on the same vertex set $S \times D$. According to Lemma 3.5. this $C_{1}$ is $r_{2}$-isomorphic to $|S| / a \cdot A$, and $\left.C_{1}\right|_{Z}$ is still isomorphic to $|S| \cdot D$.

Next for each $\beta$-class $\rho \subset S$ we consider the subgraph $C_{1}^{\rho} \subset C_{1}$ spanned by the subset $\rho \times D$. Then $C_{1}^{\rho} \mid Z$ is $r_{3}$-isomorphic to $\left.B\right|_{Z}$, so we can enhance it with $B$. Repeating this enhancement for all $\beta$-classes we obtain a new graph $C_{2}$ on the same vertex set $S \times D$. According to Lemma 3.5, this $\left.C_{2}\right|_{X}$ is $r_{2}$-isomorphic to $|S| / a \cdot A$ and $\left.C_{2}\right|_{Y}$ is $r_{2}$-isomorphic to $|S| / b \cdot B$.

We know from Lemma 2.2 that $\left.C_{2}\right|_{X}$ is an $r_{1}$-approximation of $\operatorname{Cay}(G, X)$ and $\left.C_{2}\right|_{Y}$ is an $r_{1}$-approximation of $\operatorname{Cay}(G, Y)$. Let $\gamma$ be a simple cycle of length at most $2 r+1$ of $C_{2}$. Then by Lemma 3.3, $\gamma$ lies entirely in a single $\alpha$-class or it lies entirely in a single $\beta$-class. According to Claim 3.7. $C_{2}$ is an $r$-approximation of $\operatorname{Cay}\left(G *_{\phi} H, X \cup Y\right)$. Hence the lemma follows.

Proof of the main theorem. Let $G, H$ be arbitrary sofic groups, $\Gamma$ be an amenable subgroup of $G$ and let $\phi: \Gamma \rightarrow H$ be an injective homomorphism. Then the 
amalgamated product $G \star_{\phi} H$ is the direct limit of amalgamated products $\left\{G_{\alpha} \star_{\phi} H_{\alpha}\right\}$ over amenable subgroups $\Gamma_{\alpha}$, where $G_{\alpha}, H_{\alpha}$ and $\Gamma_{\alpha}$ are all finitely generated groups. Since the direct limits of sofic groups are sofic, $G \star_{\phi} H$ is a sofic group.

\section{Hyperfiniteness}

The notion of hyperfinite graph classes was introduced in [3]. A sofic approximation $\left\{G_{n}\right\}_{n=1}^{\infty}$ is hyperfinite if for any $\epsilon>0$ there exists $K_{\epsilon}>0$ such that for any $n \geq 1$ one can erase $\epsilon\left|E\left(G_{n}\right)\right|$ edges of $G_{n}$ to obtain a graph $G_{n}^{\prime}$ having components of size not greater than $K_{\epsilon}$.

Proposition 4.1. Let $\left\{G_{n}\right\}_{n=1}^{\infty}$ be a sofic approximation of the Cayley graph of a finitely generated group $\Gamma$. Then $\left\{G_{n}\right\}_{n=1}^{\infty}$ is hyperfinite if and only if $\Gamma$ is amenable.

Proof. Let $\Gamma$ be amenable. Then $\left\{G_{n}\right\}_{n=1}^{\infty}$ is hyperfinite by Proposition 2.8 So let us suppose that $\Gamma$ is nonamenable and let $S$ be a symmetric generating system of $\Gamma$. The isoperimetric constant of $\operatorname{Cay}(\Gamma, S)$ is defined as

$$
i(\operatorname{Cay}(\Gamma, S)):=\inf _{A \subset \Gamma,|A|<\infty} \frac{|L(A)|}{|A|},
$$

where $L(A)$ is the set of edges having one endpoint in $A$ and another endpoint in $A^{c}$. It is well known that $i(\operatorname{Cay}(\Gamma, S))=c>0$. Now let $\left\{G_{n}\right\}_{n=1}^{\infty}$ be a sofic approximation of $\operatorname{Cay}(\Gamma, S)$ and suppose that $\left\{G_{n}\right\}_{n=1}^{\infty}$ is hyperfinite. Let $\left\{G_{n}^{\prime}\right\}_{n=1}^{\infty}$ be a graph sequence having components of size at most $K$ such that each $G_{n}^{\prime}$ is obtained by erasing $\frac{c}{10}\left|E\left(G_{n}\right)\right|$ edges from $G_{n}$. Observe that at least half of the vertices of $G_{n}$ are in such a component $A$ of $G_{n}^{\prime}$ such that

$$
\frac{\left|L_{G_{n}}(A)\right|}{|A|} \leq \frac{c}{10}
$$

Notice that the $K$-neighborhood of such vertices cannot be isomorphic to the $K$ neighborhood of the unit element in $\operatorname{Cay}(\Gamma, S)$. This is in contradiction with the assumption that $\left\{G_{n}\right\}_{n=1}^{\infty}$ is a sofic approximation.

\section{The proof of Theorem 2}

Let $\psi, \phi: \Gamma \rightarrow \Sigma$ be conjugate faithful embeddings of the group $\Gamma$. Let $[\psi],[\phi]$ : $\Gamma \rightarrow \prod_{n=1}^{\infty} S_{n}$ be representatives of the maps $\psi$, resp. $\phi$. This gives us for each $n \geq 1$ the $S$-colored graphs $G_{n}$ and $H_{n}$ on the set $\{1,2, \ldots, n\}$.

By the conjugacy assumption, for any $r \geq 1$ there exist subsequences $\left\{G_{n_{k}}\right\}_{k=1}^{\infty},\left\{H_{n_{k}}\right\}_{k=1}^{\infty}$ such that

- For any $k \geq 1, G_{n_{k}}$ and $H_{n_{k}}$ are $r$-isomorphic to each other.

- Both $G_{n_{k}}$ and $H_{n_{k}}$ are $r$-approximations of $\operatorname{Cay}(\Gamma, S)$.

Now let $\left\{G_{n}\right\}_{n=1}^{\infty}$ be a sofic approximation of $\operatorname{Cay}(\Gamma, S)$ such that $\left|V\left(G_{n}\right)\right|=n$ and $\Gamma$ is nonamenable. By Proposition 4.1, we can suppose that there exists $\epsilon>0$ and a function $r: \mathbb{N} \rightarrow \mathbb{N}$ tending to infinity such that if we erase $\epsilon\left|E\left(G_{n}\right)\right|$ edges from $G_{n}$, then at least one of the remaining components has size at least $r(n)$. Now consider the sofic approximation $\left\{H_{n}\right\}_{n=1}^{\infty}$, where $H_{n}$ consists of $\left[\frac{n}{r(n)}\right]$ disjoint copies of $G_{r_{n}}$ and $n-\left[\frac{n}{r(n)}\right]$ single vertices. Let $\psi$ resp. $\phi: \Gamma \rightarrow \Sigma$ be the embeddings associated to the sofic approximations $\left\{G_{n}\right\}_{n=1}^{\infty}$ resp. $\left\{H_{n}\right\}_{n=1}^{\infty}$. If $\psi$ and $\phi$ are conjugate, then by the previous arguments there exists $n_{k}>0$ such that $G_{n_{k}}$ and 
$H_{n_{k}}$ are $\left[\frac{1}{2 \epsilon}\right]$-isomorphic to each other. Therefore, one can erase $\epsilon\left|E\left(G_{n_{k}}\right)\right|$ edges from $G_{n_{k}}$ to obtain a graph having components of size at most $r\left(n_{k}\right)$, leading to a contradiction. Thus there exist $\phi, \psi: \Gamma \rightarrow \Sigma$ nonconjugate faithful embeddings provided that $\Gamma$ is nonamenable.

Now let $\Gamma$ be amenable and $\psi, \phi: \Gamma \rightarrow \Sigma$ be embeddings of $\Gamma$. Let $\left\{G_{n}\right\}_{n=1}^{\infty}$ and $\left\{H_{n}\right\}_{n=1}^{\infty}$ be the associated $S$-graphs (for representatives $[\psi]$ and $[\phi]$ ). For $n \geq 1$, let $h(n)$ be the largest integer for which $G_{n}$ and $H_{n}$ are both $h(n)$-approximations of $\operatorname{Cay}(\Gamma, S)$. Using the definition of the ultraproduct it is easy to see that for any $t \geq 1$,

$$
A_{t}=\{n \mid h(n) \geq t\} \in \omega,
$$

where $\omega$ is a nonprincipal ultrafilter. By Proposition 2.8, there exists a sofic approximation $\left\{J_{n}\right\}_{n=1}^{\infty}$ such that:

- $\left|V\left(J_{n}\right)\right|=n$.

- $G_{n}$ and $J_{n}$, resp. $H_{n}$ and $J_{n}$, are $2 r$-isomorphic whenever $h(n)$ is large enough.

Therefore $G_{n}$ and $H_{n}$ are $r$-isomorphic if $h(n)$ is large enough. Now we construct elements $g \in \prod_{\omega} S_{n}$ such that $g \psi g^{-1}=\phi$. For each $n \geq 1$, let $l(n)$ be the largest integer such that $G_{n}$ and $H_{n}$ are $l(n)$-isomorphic. Let $g_{n} \in S_{n}$ be a bijection such that $g_{n}\left(G_{n}^{\prime}\right)=H_{n}^{\prime}$, where $G_{n}^{\prime}$ resp $H_{n}^{\prime}$ are isomorphic subgraphs obtained by erasing $\frac{1}{l_{n}}\left|E\left(G_{n}\right)\right|$ resp. $\frac{1}{l_{n}}\left|E\left(H_{n}\right)\right|$ edges from $G_{n}$ resp. $H_{n}$. Then obviously $g \psi g^{-1}=\phi$.

\section{REFERENCES}

[1] B. Collins and K. J. Dykema, Free products of sofic groups with amalgamation over monotileabe amenable groups, to appear in Münster J. of Math.

[2] G. Elek, The strong approximation conjecture holds for amenable groups, Journal of Functional Analysis, 239 (2006) 345-355. MR:2258227 (2007m:43001)

[3] G. Elek, The combinatorial cost, l'Enseignement Math. 53 (2007) 225-236. MR2455943 (2009m:05083)

[4] G. Elek and E. Szabó, On sofic groups, Journal of Group Theory 9 (2006), no. 2, 161-171. MR.2220572 (2007a:20037)

[5] G. Elek and E. Szabó, Hyperlinearity, essentially free actions and $L^{2}$-invariants. The sofic property, Math. Ann. 332 (2005) 421-441. MR2178069 (2007i:43002)

[6] Kenley Jung, Amenability, tubularity, and embeddings into $R^{\omega}$, Math. Ann. 338 (2007), no. 1, 241-248. MR2295511 (2009h:46113)

[7] L. Paunescu, On sofic actions and equivalence relations (preprint), http://arxiv.org/ pdf/1002.0605.

[8] V. Pestov, Hyperlinear and sofic groups: a brief guide, Bull. Symbolic Logic 14 (2008), no. 4, 449-480. MR2460675 (2009k:20103)

Alfred Renyi Mathematical Institute, Hungarian Academy of Science, P.O. Box 127, BudAPEST, 1364, Hungary

Alfred Renyi Mathematical Institute, Hungarian Academy of Science, P.O. Box 127, BudAPEST, 1364, Hungary 